\title{
Effective Site for the Application of Extracorporeal Shock-Wave Therapy on Spasticity in Chronic Stroke: Muscle Belly or Myotendinous Junction
}

\author{
Sang Ho Yoon, MD, Min Kyung Shin, MD, Eun Jung Choi, MD, Hyo Jung Kang, MD
}

Department of Physical Medicine and Rehabilitation, Veterans Health Service Medical Center, Seoul, Korea

\begin{abstract}
Objective To compare the effect of extracorporeal shock-wave therapy (ESWT) applied at the muscle belly and myotendinous junction on spasticity in the upper and lower limbs of chronic stroke patients.

Methods Of the 151 patients, a total of 80 patients with stroke-induced spasticity on the elbow flexor and 44 patients on the knee flexor were enrolled for a prospective, randomized clinical trial. The patients were divided into control, muscle belly, and myotendinous junction groups, and a total of three ESWT sessions (0.068-0.093 $\mathrm{mJ} / \mathrm{mm}^{2}, 1,500$ shots) were conducted at one per week. A Modified Ashworth Scale (MAS) and Modified Tardieu Scale (MTS) were collected at the baseline and at 1 week after each session.

Results After interventions, the MAS and MTS of both the belly and the junction groups showed positive effects from the ESWT on spasticity in the elbow and knee flexors, but the control group did not. The results also tended to improve after each session until the entire intervention was completed. However, there was no significant difference between the belly and junction groups.

Conclusion ESWT could be effective for treating chronic spasticity after stroke when applied to muscle belly or myotendinous junction.
\end{abstract}

Keywords High-energy shock waves, Muscle spasticity, Stroke

\section{INTRODUCTION}

According to Lance [1], spasticity is commonly defined as a disorder of the sensorimotor system, characterized by a velocity-dependent increase in tonic stretch reflexes ('muscle tone') and exaggerated tendon jerks, as a result of hyperexcitability of the stretch reflex; moreover, spasticity is typical of upper motor neuron syndrome.

Received June 20, 2016; Accepted October 10, 2016

Corresponding author: Hyo Jung Kang

Department of Physical Medicine and Rehabilitation, Veterans Health Service Medical Center, 53 Jinhwangdo-ro 61-gil, Gangdong-gu, Seoul 05368, Korea. Tel: +82-2-2225-1399, Fax: +82-2-2225-1579, E-mail: khojing@hanmail.net

ORCID: Sang Ho Yoon (https://orcid.org/0000-0001-8582-9966); Min Kyung Shin (http://orcid.org/0000-0003-1184-9715); Eun Jung Choi (http:// orcid.org/0000-0001-7894-1730); Hyo Jung Kang (http://orcid.org/0000-0002-8493-3160).

(c) This is an open-access article distributed under the terms of the Creative Commons Attribution Non-Commercial License (http://creativecommons.org/ licenses/by-nc/4.0) which permits unrestricted noncommercial use, distribution, and reproduction in any medium, provided the original work is properly cited. Copyright (c) 2017 by Korean Academy of Rehabilitation Medicine 
The pathophysiological mechanisms of spasticity involve abnormalities in intraspinal processing, which usually regulates the excitatory and inhibitory signals in the intraspinal circuit, as well as imbalances in descending regulations, which modulate the excitatory and inhibitory descending inputs from the supraspinal roots [2].

Spasticity is a common complication after stroke; onset may range from a few days to 18 months [3]. The prevalence of spasticity ranges from $19 \%$ to $92 \%$ overall, and may reach up to $42.6 \%$ at 6 months after stroke $[2,4]$. Although spasticity has positive effects, such as functional support of gait or standing in stroke patients, it also causes problems such as limitations of joint range of movement, difficulty in daily activities, pain, caregiver burden, and increase in treatment cost [5,6]. Approximately $4 \%$ of patients with severe spasticity require interventions [3]. The management of spasticity includes treatments such as range of motion exercises focusing on stretching, physical modalities, oral medications, intrathecal infusion of medications, and surgical interventions, including muscle tendon transfer [6]. Extracorporeal shock-wave therapy (ESWT) has recently been applied as a noninvasive therapy for spasticity after stroke.

A shock wave is a sequence of single acoustic pulses, characterized by a high peak pressure, rapid pressure increase, short duration of application, and rapid propagation in three-dimensional spaces [7]. The principles of shock-wave therapy are based on tensile forces in the negative phase, which generate cavitation bubbles, shear forces that are called a 'jet stream,' and the reflection of positive pressure that directly transfers energy to the target tissue [8,9]. Several studies have shown that ESWT is effective for treating spasticity after stroke $[7,10]$. In a meta-analysis of five studies, Lee et al. [10] assessed the effect of ESWT in cases of spasticity after brain injury, and found a significant improvement (compared to the baseline values) in the Modified Ashworth Scale (MAS) after four ESWT sessions. In addition, Dymarek et al. [7] reviewed articles on the antispasticity effect of ESWT over the last 10 years. Of 91 clinical articles identified, 8 showed relevant effects of ESWT on spasticity in the upper and lower extremities. However, only one randomized, controlled study, evaluated via the Cochrane method, was on a treatment group with 5 ESWT sessions on the finger flexor muscles; the treated group showed much more improvements in the MAS grade than the control group did. ESWT may therefore be a safe treatment for cases of spasticity after stroke.

Although previous studies have assessed the effect of ESWT on spasticity, only a few reports have described how to maximize the effect of ESWT on the muscles. In an in vivo study on healthy rats, Kenmoku et al. [11] reported that ESWT led to the degeneration and reduction in the number of acetylcholine receptors in the neuromuscular junctions, which suggests that it may decrease spasticity. ESWT is usually applied on the muscle belly [7].

Because ESWT is based on the penetration of single acoustic pulses into a limited area, it is important to know exactly how to apply it [7]. Despite this, there was only one study that focused on the ESWT application sites [12]. Bae et al. [12] compared the effect of ESWT applied at the muscle belly and at the myotendinous junction, given that the Ib afferent fiber of the Golgi tendon in the myotendinous junction inhibits $\alpha$ motor neurons; they found that treatment at the myotendinous junction was more effective than that at the muscle belly. However, in that study, only a few patients were enrolled in each group (muscle belly, $\mathrm{n}=12$; myotendinous junction, $\mathrm{n}=11$ ), the ESWT was applied to only one muscle of the biceps brachii, and only two groups were compared. They emphasized that further study was needed to supplement their study, in order to have more reliable results about the effect of ESWT applied at the muscle belly and at the myotendinous junction on spasticity in stroke patients.

In our study, we aimed to compare the effects of ESWT applied at the muscle belly and at the myotendinous junction on spasticity in the upper and lower limbs of chronic stroke patients.

\section{MATERIALS AND METHODS}

\section{Subjects}

From May 2015 to March 2016, 151 chronic stroke patients who were hospitalized at the Department of Physical Medicine and Rehabilitation at Veterans Health Service Medical Center were recruited for a prospective, randomized control study. The inclusion criteria were as follows: stroke as confirmed by computed tomography or magnetic resonance imaging, duration of at least 6 months since stroke onset, and an MAS grade of $>1+$ at the elbow (biceps brachii) and knee flexor (semiten- 
dinosus muscle) $[13,14]$. The exclusion criteria were as follows: joint contracture, history of botulinum toxin or alcohol injection, and severe medical problems or orthopedic disease. Before we began the shock-wave treatments, the antispasticity medication and physical therapy for spasticity received by the patients were noted and the notes were maintained throughout the study. Of the 151 patients, a total of 80 were assigned to the elbow flexor group, 44 to the knee flexor group. Thereafter, for treatment, the patients were randomly allocated to the control group (no ESWT application), muscle belly group (Belly group; ESWT applied at the muscle belly), or myotendinous junction group (Junction group; ESWT applied at the myotendinous junction) by block randomization (Fig. 1). Our study was approved by the Institutional Review Board (No. 2015-03-025-002), and written informed consent was acquired from all patients.

\section{ESWT procedure}

The depth and location of the muscle belly and the myotendinous junction in the biceps brachii and semitendinosus muscles were confirmed by using ultrasonography (US) (Accuvix XQ; Medison, Seoul, Korea). In the biceps brachii, the muscle belly was described as the bulk of the muscle in the mid-arm, whereas the myotendinous junction was described as the point where the tendon aponeurosis was located centrally within the muscle, based on US findings. In a semitendinosus muscle, such as the knee flexor, the muscle belly could be easily detected because its location is superficial relative to the other medial hamstring muscles. In fact, the muscle belly was confirmed as the thickest portion at the site of superficial bulk on the muscle in the medial hamstrings, based on US findings. The myotendinous junction was also confirmed using US. One researcher (SH Yoon) evaluated the muscle belly and myotendinous junction sites by using US.

After marking the sites, ESWT (Dornier Aries; Dornier MedTech, Wessling, Germany) was applied at the elbow and knee flexors in the patients from the three groups. All the patients completed three ESWT sessions at each site, once every week at the same time. Patients were comfortably placed in the supine position at the elbow flexor and in the prone position at the knee flexor during the intervention. In the belly and junction groups, we applied stimulation with the following parameters: energy, $0.068-0.093 \mathrm{~mJ} / \mathrm{mm}^{2}$; frequency, $5 \mathrm{~Hz}$; and number of shots, 1,500 times within tolerable pain limits $[7,10]$. The control group received only sound over the biceps brachii and semitendinosus muscles without any transducer contact.

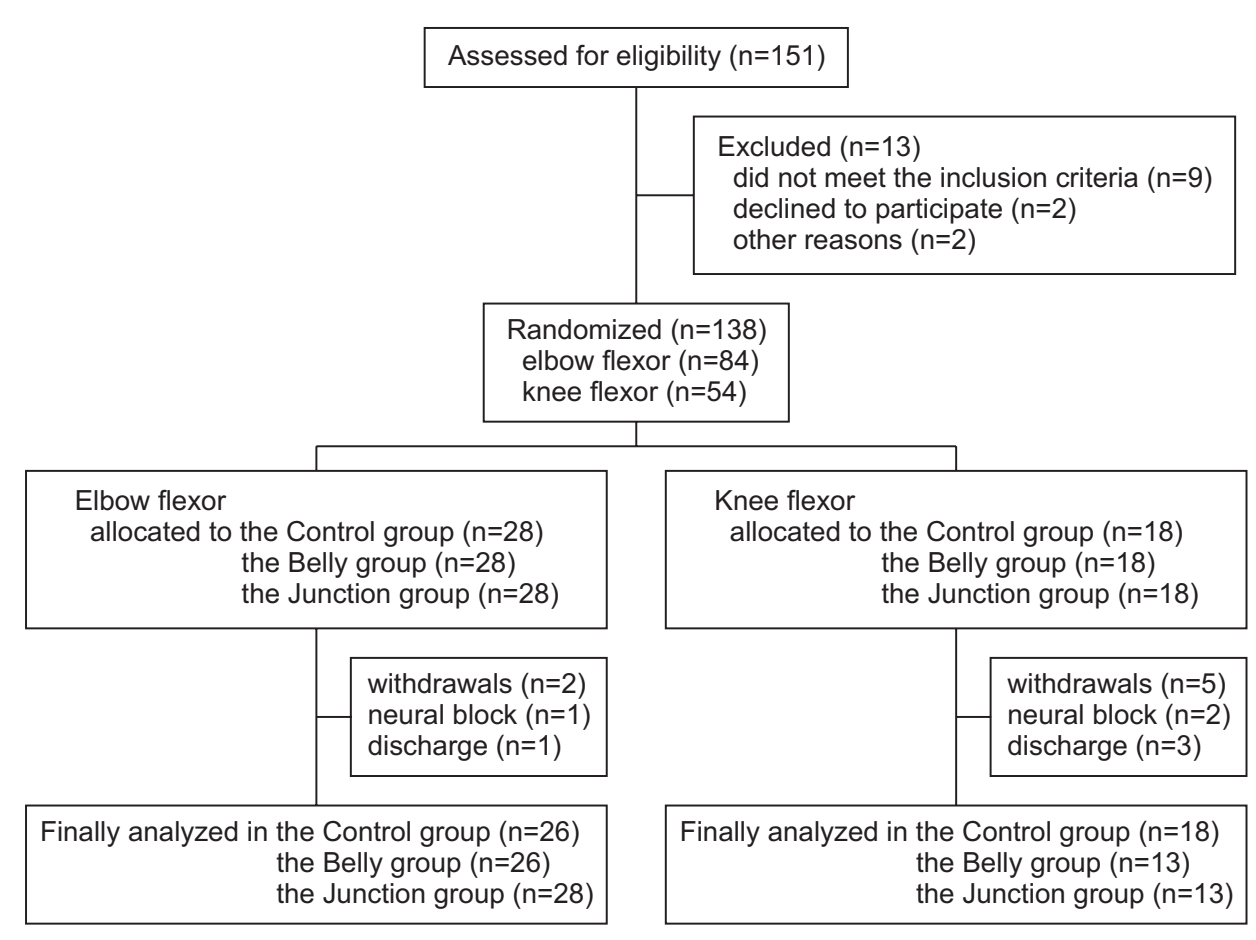

Fig. 1. Flowchart of the study participants. Control group, no extracorporeal shock wave therapy (ESWT) application; belly group, ESWT applied at the muscle belly; junction group, ESWT applied at the myotendinous junction. 


\section{Outcome measurements}

The MAS and Modified Tardieu Scale (MTS) were measured by a single physician (SH Yoon) to evaluate the spasticity of the biceps brachii and semitendinosus muscles. The biceps brachii is responsible for forearm supination. Therefore, we measured the MAS and MTS values of the elbow flexor with pronation of the forearm to exclude any interference. Furthermore, knee flexion could be affected by the gastrocnemius muscle, which is responsible for knee flexion and ankle plantar flexion. Hence, in order to measure the MAS and MTS values of the semitendinosus muscle, the patient was placed in the prone position, and the ankle was in the neutral position, to avoid an effect by the gastrocnemius muscle during the measurements. MAS is a manual measurement of the resistance of muscles during passive stretching [13]. MTS is used to measure the resistance during a rapid passive stretch $[14,15]$. For statistical compliance, MAS 1+ was equivalent to scores of $2,2-3,3-4$, and 4-5. In this study, the MTS was used to assess the range of movement during a quick stretch and was measured using a digital goniometer. The MTS value was recorded at the catch angle when the elbow and knee were extended from maximal flexion. The angle was evaluated 3 times, and the average value was recorded. Baseline evaluations were conducted before the start of treatment, and the outcome measures were evaluated at 1 week after each session in all the groups, for a total of 4 evaluations.

\section{Sample size}

The G*Power program (v3.1.9.2; http://www.gpower. hhu.de/) was used to identify a suitable sample size for the intervention. For the elbow flexor group, the input parameters were: mean of difference, 3.2; standard deviation of difference, 10.1 ; $\alpha$-error, 0.05 ; power, $80 \%$; effect size, 0.31 ; number of measurements, 3 ; and nonsphericity correction $\varepsilon, 0.5$. For the knee flexor group, the input parameters were: mean of difference, 4.7 ; standard deviation of difference, 9.3 ; $\alpha$-error, 0.05 ; power, $80 \%$; effect size, 0.5 . The mean and standard deviation values were calculated by our previous pilot studies. The results showed that 28 patients each were required for the elbow flexor groups, whereas 12 patients each were required for the knee flexor groups. Hence, a total of 138 patients were enrolled, to allow for possible drop-outs.

\section{Statistical analysis}

Statistical Package for Social Sciences (SPSS) software ver. 18.0 (SPSS Inc., Chicago, IL, USA) was used for statistical analysis. The general characteristics were evaluated using one-way analysis of variance or Kruskal-Wallis test. The effects between pre- and post-intervention were compared by using a paired t-test or a Wilcoxon signedrank test within the groups. For comparisons with the control group, an independent t-test or Mann-Whitney U-test was used. Repeated-measures analysis of variance (RM ANOVA) or a Wilcoxon signed-rank test was used to compare the significant differences after each session until treatment was completed for each group. A p-value of $<0.05$ was considered to be statistically significant.

\section{RESULTS}

\section{Baseline characteristics}

The participants' general characteristics are presented in Table 1. There were no significant differences in age, stroke duration, height, Modified Barthel Index (MBI), manual muscle test (MMT), MAS grades, and MTS, except for body mass index in the elbow flexor group.

\section{Effects of ESWT on spasticity after all 3 sessions in each group}

There were significant improvements in the MAS and MTS values for the belly and junction groups at both the elbow and knee flexors; however, there was no significant change in the control group before and after treatment (Table 2). The belly and junction groups both showed positive effects of ESWT on spasticity in terms of the MAS and MTS, whereas the control group did not, at the elbow and knee flexors (Fig. 2). However, there was no significant difference between the belly and junction groups in terms of the MTS value at the elbow ( $\mathrm{p}=0.748$, independent t-test) and knee flexors ( $p=0.103$, Mann-Whitney Utest) (Fig. 2).

\section{Effects of ESWT on spasticity after each session At the elbow flexor}

The MAS grade significantly differed between the baseline and the second session, and between the baseline and the third session, in both the belly and the junction groups; in particular, the difference in the MAS grade was marked between the first session and the second session, 


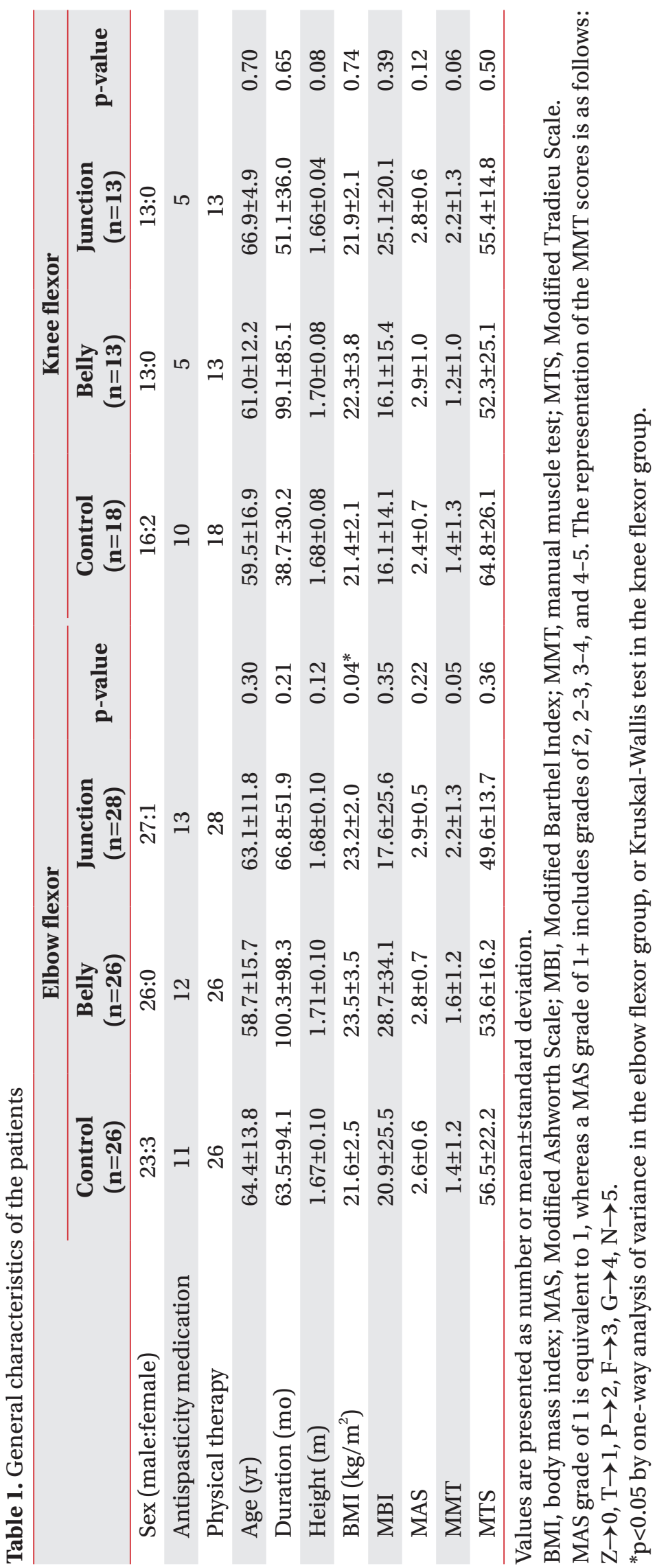


Sang Ho Yoon, et al.

Table 2. Comparison of MAS and MTS before and after the 3 ESWT sessions at the elbow and knee flexors

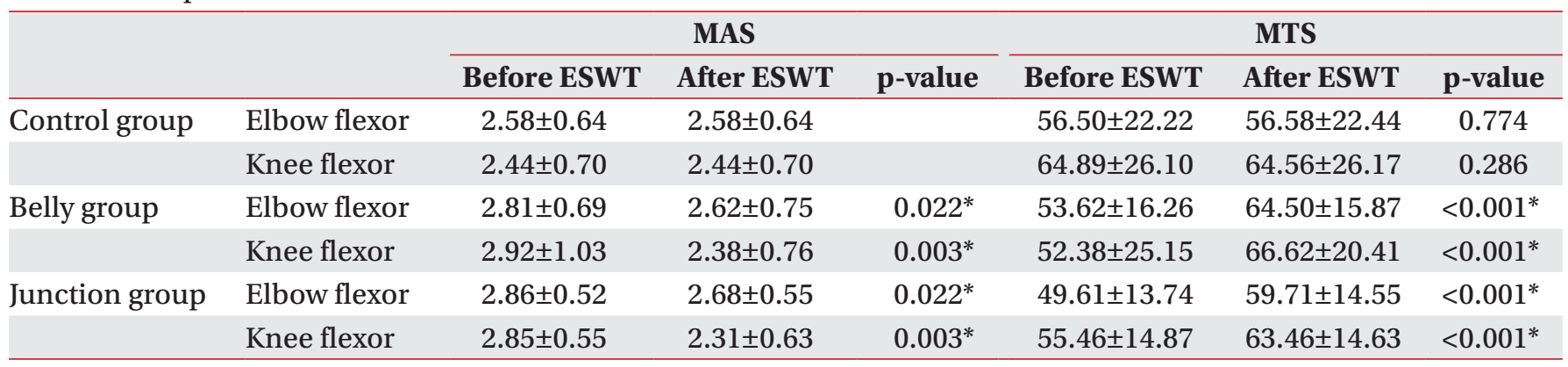

Values are presented as mean \pm standard deviation.

ESWT, extracorporeal shock wave therapy; MAS, Modified Ashworth Scale; MTS, Modified Tradieu Scale. ${ }^{*} \mathrm{p}<0.05$ by paired $\mathrm{t}$-test in the elbow flexor group or Wilcoxon signed rank test in the knee flexor group.

(A)

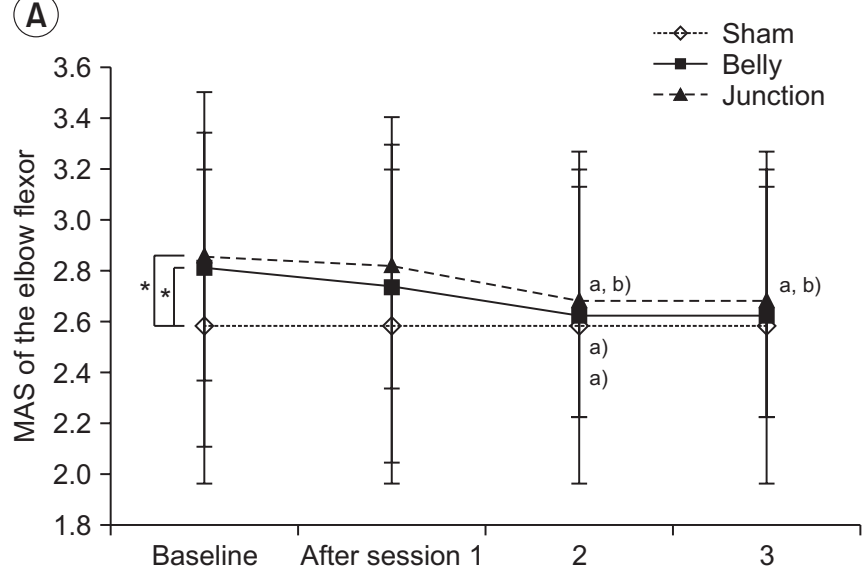

(C)

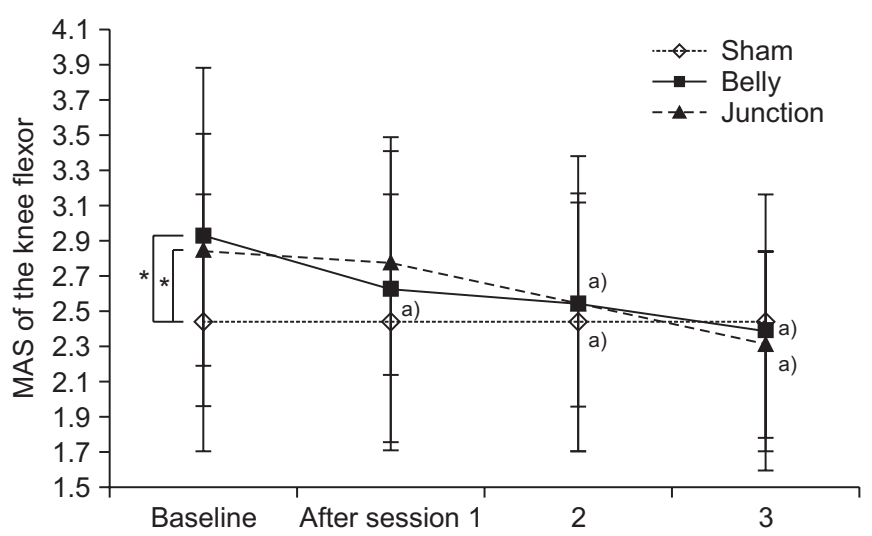

(B)

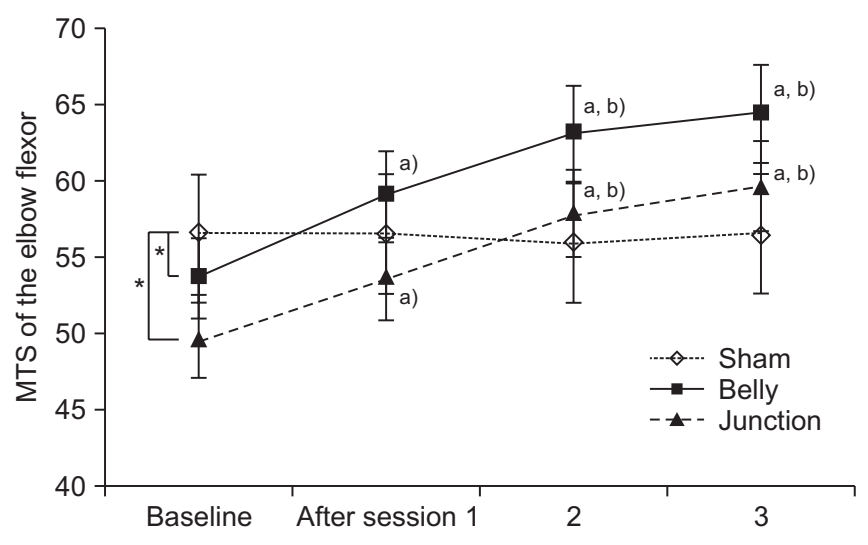

(D)

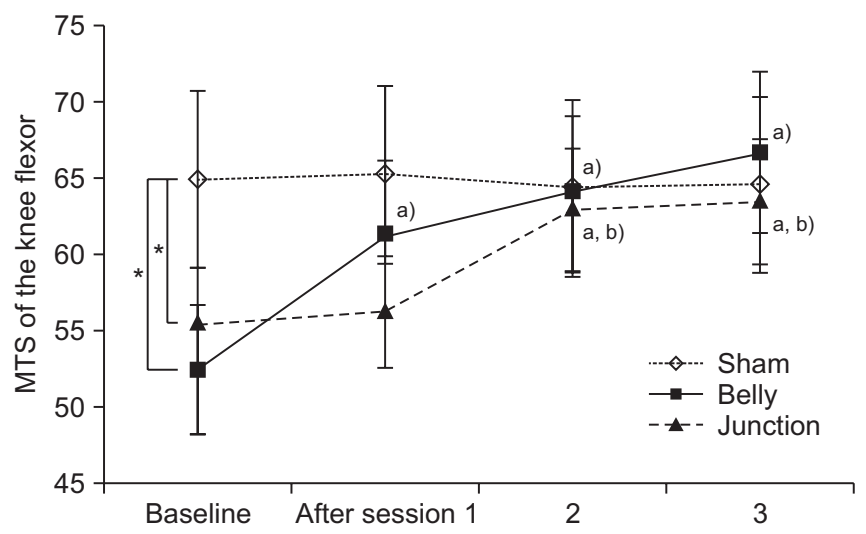

Fig. 2. Effects of ESWT in the elbow flexor and knee flexor. (A, B) MAS and MTS in the elbow flexor group, (C, D) MAS and MTS in the knee flexor group. ${ }^{*} \mathrm{p}<0.05$, compared to the control group using an independent t-test or MannWhitney U-test. ${ }^{\text {a) }} \mathrm{p}<0.05$, compared to the baseline values using repeated-measures analysis of variance or Wilcoxon signed-rank test with Bonferroni correction within each group. ${ }^{\text {b) }} \mathrm{p}<0.05$, compared to the value after session 1 using repeated-measures analysis of variance or Wilcoxon signed-rank test with Bonferroni correction within each group. ESWT, extracorporeal shock wave therapy; MAS, Modified Ashworth Scale; MTS, Modified Tradieu Scale. 
and between the first session and the third session, in the junction group (Fig. 2A). The MTS had significantly improved during the first, second, and third sessions, as compared to that at baseline in both the belly and junction groups; moreover, a significant improvement was observed between the first session and the second session, and between the first session and the third session, in both the belly and the junction groups based on RM ANOVA (Fig. 2B). Thus, the effects of ESWT seem to appear immediately after the first session and are maintained throughout the remainder of the sessions (Fig. 2B).

\section{At the knee flexor}

The MAS grades significantly differed during the first, second, and third sessions, as compared to that at the baseline in the belly group, and also significantly differed between the baseline and the second session, and between the baseline and the third session, in the junction group, based on the Wilcoxon signed-rank test (Fig. 2C). Moreover, the MTS values significantly improved during the first, second, and third sessions, as compared to that at the baseline in the belly group, and significantly improved from the baseline to the second and third sessions and from the first session to the second and third sessions in the junction group (Fig. 2D). These results also tended to improve after each session until the overall treatment was completed.

\section{DISCUSSION}

In this study, we sought to compare the effects of ESWT, applied at the belly or myotendinous junction, on the spasticity induced after stroke at the elbow and knee flexors in chronic patients. There was no significant difference in the MAS and MTS values between the belly and junction groups in both limbs. However, ESWT showed significant effects on spasticity at both sites, and progressive improvements were noted as the ESWT sessions were continued.

Several studies have described the effects of ESWT on stroke-induced spasticity $[7,12,16-18]$. Yoo et al. [16] reported that the stimulation of 21 chronic stroke patients with elbow flexor and wrist pronator spasticity led to significant improvements after 1 week, which persisted for at least 4 weeks. Moreover, the spasticity reduced as the number of sessions was increased. Sohn et al. [17] stimulated the medial head of the gastrocnemius during 1 session $\left(0.1 \mathrm{~mJ} / \mathrm{mm}^{2}\right.$ and 1,500 shots $)$ in chronic stroke patients, and found that the MAS of the ankle plantar flexor was significantly reduced. Moon et al. [18] also stimulated the musculotendinous junction of the medial and lateral gastrocnemius three times $\left(0.089 \mathrm{~mJ} / \mathrm{mm}^{2}\right.$, $4 \mathrm{~Hz}$, and 1,500 shots) in subacute stroke patients, and found that the ankle plantar flexor spasticity had significantly improved for at least a week after ESWT, but was not maintained for 4 weeks. The findings of this study are consistent with the abovementioned results. Although many previous studies have evaluated the effect of ESWT on spasticity, only a few studies have assessed the use of different application sites for obtaining better outcomes. One advantage of our randomized controlled study was the application of ESWT at sites of the muscle belly and myotendinous junction in both the upper and lower extremities, along with a control group.

The Golgi tendon in the myotendinous junction affects spasticity during the inverse stretch reflex via the Ib afferent fiber [2]. Accordingly, we hypothesized that the effects of ESWT on spasticity may depend on the application site (muscle belly or myotendinous junction). In a study of 12 patients who underwent ESWT at the muscle belly and 11 patients who underwent ESWT at the myotendinous junction, Bae et al. [12] reported that ESWT at the myotendinous junction yielded better results than ESWT at the muscle belly. In our study, we examined more patients (a total of 80 in the elbow flexor and 44 in the knee flexor muscles), performed ESWT at the upper and lower extremities, and also compared the results with a control group to improve the reliability. However, we did not observe a significant difference in the effects of ESWT between the muscle belly and myotendinous junction for both the elbow and knee flexor after treatment.

These findings may be associated with the mechanism of ESWT. Of several reported mechanisms underlying the effect of ESWT, the most widely reported is that shock waves induce the synthesis of nitric oxide, which plays major roles in vasodilatation, neoangiogenesis, increase of blood flow, and regulation of inflammation in many musculoskeletal diseases (plantar fasciitis, myofascial pain syndrome, and calcific tendinitis) [19-21]. Although the manner in which shock waves reduce spasticity remains unclear, previous studies $[10,22]$ have suggested 
that the synthesis of nitric oxide reduces spasticity by means of an anti-inflammatory effect and by serving as a transmitter in the nervous system. Shock waves also alleviate the passive stiffness of muscles caused by connective tissue inactivity, and can affect muscle fibers around the tendon via direct mechanical stimuli. Most of the patients enrolled in this study were at a chronic stage after stroke (Table 1). Spasticity in chronic patients could immobilize paralyzed muscles, which could consequently lead to soft tissue rearrangements, shortening of the muscles, and joint and soft tissue contracture. In addition, changes in muscle properties could also appear [2]. Moreover, in chronic patients, ESWT may work by improving connective tissue stiffness and spastic muscle fibrosis [7]. These findings were noted in both the muscle belly and myotendinous junction groups.

In addition, the finding that the MTS value of the elbow flexor showed significant improvements after even the first session may support the abovementioned reasoning. The improvements in the MTS value persisted throughout the sessions in both the belly and junction groups (Fig. 2B). Moreover, the MTS value of the knee flexor had significantly improved after the first session in the belly group and the second session in the junction group (Fig. 2D). The difference in the findings between the elbow and knee flexors might be explained as follows. Elbow flexion is primarily mediated by the biceps brachii and brachialis; in contrast, knee flexion is mediated through the activation of the semimembranosus, semitendinosus, biceps femoris, sartorius, popliteus, gastrocnemius, and gracilis muscles [23]. In this study, we focused on the semitendinosus muscles in the knee flexors, although other muscles would still affect knee flexor spasticity. Very few studies have evaluated the effect of ESWT at each session on spasticity in chronic stroke patients. To our knowledge, this is the first study to evaluate the effect of ESWT for treating the stroke-induced spasticity in the upper and lower extremities evaluated after each session during the intervention period.

This study has certain limitations. First, subjects with stroke were at a chronic stage; for most, it had been more than 60 months since the onset. Hence, spasticity in acute or subacute stroke patients was not evaluated. Second, there were not enough participants for us to compare patients in the knee flexor group and elbow flexor group. There may be different results for the upper and lower limbs, depending on the application sites, as well as different muscle features. Third, the follow-up interval was only a week. According to other studies $[16,22]$, the effect of ESWT is maintained for at least 4 and up to 12 weeks after treatment; hence, it is essential to assess how long the effect of ESWT persists for patients whose stroke occurred more than 5 years ago. Fourth, although our study was prospective, most of the patients enrolled were men. Hence, there is a need to evaluate whether the effect of ESWT for spasticity in women will show results similar to those for men.

However, this study also has strengths. First, it focused on the application site of ESWT on spastic muscles in upper and lower limbs. Although there have been many studies about the effect of ESWT on spasticity, few studies have evaluated the specific application sites. Second, this study attempted to compare the muscle belly and myotendinous junction sites with a control therapy group, which made this study more reliable than previous ones. Third, this study evaluated the effect of ESWT after each session. As a result, the serial effects of ESWT on spasticity were observed throughout the intervention period.

In this study, we compared the effects of ESWT, applied at the belly or myotendinous junction, on the spasticity induced after stroke at the elbow and knee flexors in chronic patients. The current study suggests that ESWT could be effective for treating spasticity after stroke, no matter which application site (muscle belly or myotendinous junction) is used. However, further studies are needed to establish how long the effect of ESWT on chronic stroke patients is maintained.

\section{CONFLICT OF INTEREST}

No potential conflict of interest relevant to this article was reported.

\section{REFERENCES}

1. Lance JW. The control of muscle tone, reflexes, and movement: Robert Wartenberg Lecture. Neurology 1980;30:1303-13.

2. Li S, Francisco GE. New insights into the pathophysiology of post-stroke spasticity. Front Hum Neurosci 2015;9:192.

3. Sunnerhagen KS, Francisco GE. Enhancing patient- 
provider communication for long-term post-stroke spasticity management. Acta Neurol Scand 2013;128: 305-10.

4. Wissel J, Verrier M, Simpson DM, Charles D, Guinto P, Papapetropoulos S, et al. Post-stroke spasticity: predictors of early development and considerations for therapeutic intervention. PM R 2015;7:60-7.

5. Demetrios M, Gorelik A, Louie J, Brand C, Baguley IJ, Khan F. Outcomes of ambulatory rehabilitation programmes following botulinum toxin for spasticity in adults with stroke. J Rehabil Med 2014;46:730-7.

6. Santamato A, Micello MF, Ranieri M, Valeno G, Albano A, Baricich A, et al. Employment of higher doses of botulinum toxin type A to reduce spasticity after stroke. J Neurol Sci 2015;350:1-6.

7. Dymarek R, Ptaszkowski K, Słupska L, Halski T, Taradaj J, Rosinczuk J. Effects of extracorporeal shock wave on upper and lower limb spasticity in post-stroke patients: a narrative review. Top Stroke Rehabil 2016; 23:293-303.

8. Romeo P, Lavanga V, Pagani D, Sansone V. Extracorporeal shock wave therapy in musculoskeletal disorders: a review. Med Princ Pract 2014;23:7-13.

9. Zelle BA, Gollwitzer H, Zlowodzki M, Buhren V. Extracorporeal shock wave therapy: current evidence. J Orthop Trauma 2010;24 Suppl 1:S66-S70.

10. Lee JY, Kim SN, Lee IS, Jung H, Lee KS, Koh SE. Effects of extracorporeal shock wave therapy on spasticity in patients after brain injury: a meta-analysis. J Phys Ther Sci 2014;26:1641-7.

11. Kenmoku T, Ochiai N, Ohtori S, Saisu T, Sasho T, Nakagawa $\mathrm{K}$, et al. Degeneration and recovery of the neuromuscular junction after application of extracorporeal shock wave therapy. J Orthop Res 2012;30:1660-5.

12. Bae H, Lee JM, Lee KH. The effects of extracorporeal shock wave therapy on spasticity in chronic stroke patients. J Korean Acad Rehabil Med 2010;34:663-9.

13. Kaya T, Karatepe AG, Gunaydin R, Koc A, Altundal Ercan U. Inter-rater reliability of the Modified Ashworth Scale and modified Modified Ashworth Scale in assessing poststroke elbow flexor spasticity. Int J Rehabil
Res 2011;34:59-64.

14. Abolhasani H, Ansari NN, Naghdi S, Mansouri K, Ghotbi N, Hasson S. Comparing the validity of the Modified Modified Ashworth Scale (MMAS) and the Modified Tardieu Scale (MTS) in the assessment of wrist flexor spasticity in patients with stroke: protocol for a neurophysiological study. BMJ Open 2012;2.

15. Boyd RN, Morris ME, Graham HK. Management of upper limb dysfunction in children with cerebral palsy: a systematic review. Eur J Neurol 2001;8 Suppl 5: 150-66.

16. Yoo SD, Kim HS, Jung PK. The effect of shock wave therapy on upper limb spasticityin the patients with stroke. J Korean Acad Rehabil Med 2008;32:406-10.

17. Sohn MK, Cho KH, Kim YJ, Hwang SL. Spasticity and electrophysiologic changes after extracorporeal shock wave therapy on gastrocnemius. Ann Rehabil Med 2011;35:599-604.

18. Moon SW, Kim JH, Jung MJ, Son S, Lee JH, Shin H, et al. The effect of extracorporeal shock wave therapy on lower limb spasticity in subacute stroke patients. Ann Rehabil Med 2013;37:461-70.

19. Mariotto S, Cavalieri E, Amelio E, Ciampa AR, de Prati AC, Marlinghaus E, et al. Extracorporeal shock waves: from lithotripsy to anti-inflammatory action by NO production. Nitric Oxide 2005;12:89-96.

20. Mariotto S, de Prati AC, Cavalieri E, Amelio E, Marlinghaus E, Suzuki H. Extracorporeal shock wave therapy in inflammatory diseases: molecular mechanism that triggers anti-inflammatory action. Curr Med Chem 2009;16:2366-72.

21. Sharma JN, Al-Omran A, Parvathy SS. Role of nitric oxide in inflammatory diseases. Inflammopharmacology 2007;15:252-9.

22. Manganotti P, Amelio E. Long-term effect of shock wave therapy on upper limb hypertonia in patients affected by stroke. Stroke 2005;36:1967-71.

23. Whitehead CL, Hillman SJ, Richardson AM, Hazlewood ME, Robb JE. The effect of simulated hamstring shortening on gait in normal subjects. Gait Posture 2007;26:90-6. 